\title{
Comparison of different ionic liquids pretreatment for barley straw enzymatic saccharification
}

\author{
Sohrab Haghighi Mood • Amir Hossein Golfeshan • \\ Meisam Tabatabaei $\cdot$ Saeed Abbasalizadeh • \\ Mehdi Ardjmand
}

Received: 20 April 2013/Accepted: 18 July 2013/Published online: 31 July 2013

(C) The Author(s) 2013. This article is published with open access at Springerlink.com

\begin{abstract}
Recently, application of ionic liquids due to their special solvency properties as a promising method of pretreatment for lignocellulosic biomass has received much attention. Chemical stability, temperature stability, nonflammability, low vapor pressure, wide liquidus range, and non-toxicity are among those unique properties. These solvents are also known as green solvents due to non-toxicity and low vapor pressure. The present study was set to compare the effect of five different ionic liquids namely, 1-ethyl-3-methyl imidazolium acetate, 1-ethyl-3-methyl imidazolium diethyl phosphate, 1-butyl-3-methyl imidazolium chlorides, 1,3-dimethyl imidazolium dimethyl phosphate, and 1-butyl-3-methylimidazolium-trifluoromethane sulfonate on barley straw in bioethanol production process. The performance of ionic liquids was evaluated based on the change observed in chemical structure, crystallinity index, and cellulose digestibility. Overall, 1-ethyl-3-methyl imidazolium acetate was found most effective in pretreating barely straw for bioethanol production. To the best of our knowledge, the present study reports different ionic liquids; some for the first time, for barely straw pretreatment.
\end{abstract}

Keywords Ionic liquid - Pretreatment - Barely straw · Crystallinity index $\cdot$ Cellulose digestibility

S. H. Mood · A. H. Golfeshan · M. Ardjmand

Department of Chemical Engineering, South Tehran Branch, Islamic Azad University, Tehran, Iran

M. Tabatabaei $(\square) \cdot$ S. Abbasalizadeh $\cdot$ M. Ardjmand Biofuel Research Team (BRTeam), Microbial Biotechnology and Biosafety Department, Agricultural Biotechnology Research Institute of Iran (ABRII), Mahdasht Road, Fahmideh Blvd., P.O. Box: 31535-1897, Karaj, Iran

e-mail: meisam_tab@yahoo.com

\section{Introduction}

Lignocellulosic biomass is suitable sources for conversion to bioethanol for they are abundant and cheap. However, conversion of lignocelluloses to bioethanol is faced with physical and chemical barriers. More specifically, crystalline structure of cellulose, presence of lignin, and covalent cross-linkages between lignin and hemicelluloses in cell wall obstruct the decomposition process of lignocellulosic materials (Haghighi Mood et al. 2013). Therefore, the goal of pretreatment is defined to overcome these obstacles including breaking down lignin structure, disrupting the crystalline structure of cellulose and cross-linked matrix of lignin and hemicelluloses, and increasing the porosity and surface area of cellulose (Alvira et al. 2010; Li et al. 2010).

To date, several pretreatment methods have been introduced including physical pretreatment (e.g. grinding and milling, microwave, and extrusion), chemical pretreatment (e.g. alkali, acid, organosolv, ozonolysis, and ionic liquid), physico-chemical pretreatment (such as steam explosion, liquid hot water, ammonia fiber explosion, wet oxidation, and $\mathrm{CO}_{2}$ explosion), and biological pretreatment. Most of the conventional pretreatment methods suffer from one or more drawbacks. For instance, dilute acid pretreatment needs costly corrosion resistant equipments and besides, leads to the production of a significant amount of fermentation inhibitors during the process (Yoon et al. 2011). Biological pretreatment also requires large space and is lengthy. Moreover, it needs continuous monitoring of microorganism growth (Wyman et al. 2005; Chandra et al. 2007). As for the organic solvents, flammability and explosion are of major concern (Galbe and Zacchi 2007). Ammonia fiber explosion, hot water, and steam explosion processes are also costly and are not yet economically feasible due to high operation cost basically due to the high 
cost of ammonia and being energy-intensive, respectively (Guragain et al. 2011). Mechanical pretreatment is energy intensive and costly as well (Haghighi Mood et al. 2013).

Recent attempts have been striving to make pretreatment methods more efficient, environmentally friendly, and cost effective (Li et al. 2009). Among them, application of ionic liquids (ILs) as a promising pretreatment method has gained much attention. ILs are organic salts composed of anions and cations and melt below $100{ }^{\circ} \mathrm{C}$ (Tan et al. 2010). The mild process condition and unique safety feature of the chemicals used in this method are regarded as the main advantages. ILs remain liquid in a wide range of temperature. Moreover, they have low vapor pressure and high chemical and thermal stability. As a result of these unique features, they are known as green solvents (Tan et al. 2010).

Depending on the selection of cations and anions to be involved in the structure of ILs, their properties (i.e. viscosity, melting point, and polarity) could be tuned (MoraPale et al. 2011). Depending on the type of ILs, they are capable of dissolving carbohydrates and lignin. In fact, hydrogen bonds are formed between the non-hydrated ions of ILs and the sugar hydroxyl protons and as a result, the complex network of cellulosic biomass polymers, hemicelluloses, and lignin is broken down (Alvira et al. 2010). The regenerated cellulose has more amorphous and porous structure than those in untreated lignocellulosic biomass. Therefore, regenerated cellulose is much more susceptible to enzymatic hydrolysis (Zhao et al. 2009). Moreover, one of the most important advantages of ILs solvents is their recyclability. More specifically, these solvent can be reused (recycled) without affecting their performance in dissolution of cellulose ( $\mathrm{Li}$ et al. 2009).

In this study, barley straw was pretreated using five different ILs, i.e. 1-ethyl-3-methyl imidazolium acetate ([EMIM][AC]), 1-ethyl-3-methyl imidazolium diethyl phosphate ([EMIM][DEP]), 1-butyl-3-methyl imidazolium chlorides ([BMIM][CL]), 1,3-dimethyl imidazolium dimethyl phosphate ([MMIM][DMP]), and 1-butyl-3-methylimidazolium-trifluoromethane sulfonate ([BMIM][OTf]). The performance of ILs was evaluated based on the change observed in chemical structure of the biomass, cellulose crystallinity index, and cellulose digestibility.

\section{Materials and methods}

Materials and preparation

Barley straw samples were collected from the research farm of Seed and Plant Improvement Institute. The straws were dried under sun before shredded into pieces. Then, the shredded straws were sieved to obtain fractions with a particle size of $0.420 \mathrm{~mm}$ and their composition, i.e. cellulose, hemicellulose, and lignin contents was determined based on National Renewable Energy Laboratory (NREL/ TP-510-42618) (Sluiter et al. 2008). The ILs 1-ethyl-3methyl imidazolium acetate, 1-ethyl-3-methyl imidazolium diethyl phosphate, 1-butyl-3-methyl imidazolium chlorides, 1,3-dimethyl imidazolium dimethyl phosphate and 1-butyl3-methylimidazolium-trifluoromethane sulfonate were purchased from Sigma-Aldrich. Cellulase from Trichoderma reesei ATCC 26921 and Cellobiase from Aspergillus niger were purchased from Sigma-Aldrich as well. The other chemicals used in this study included sulfuric acid 95-97\% (Fluka), citric acid monohydrate (Sigma), sodium hydroxide $\geq 97 \%$ (Sigma-Aldrich), 3,5-dinitrosalicylic acid $98 \%$ (Aldrich), hydrochloric acid $37 \%$ (Merck), potassium sodium tartrate tetrahydrate (Merck), sodium metabisulfite $\geq 99 \%$ (Sigma-Aldrich), Tetracycline (Sigma), and cycloheximide $\geq 93 \%$ (Fluka).

\section{Barley straw pretreatment}

A $4 \%(w / w)$ barley straw solution or in other words, a $96 \%$ IL solution was prepared by combining $200 \mathrm{mg}$ of barley straw with $4.8 \mathrm{~g}$ IL in a test tube. The test tubes containing the samples were stirred $(150 \mathrm{rpm})$ and heated in an oil bath at $110{ }^{\circ} \mathrm{C}$ for $90 \mathrm{~min}$. All experiments were carried out in triplicates. After $90 \mathrm{~min}$ of incubation, the reaction mixtures were cooled down to $60{ }^{\circ} \mathrm{C}$ and then $50 \mathrm{ml}$ deionized water as an anti solvent was added to precipitate and regenerate the dissolved cellulose. Next, the precipitated material was filtered through filtering paper (Whatman No. 2) using Buchner funnel under a reduced pressure and washed with deionized water in order to ensure that excess ionic liquid had been removed. Then prior to enzymatic hydrolysis, the precipitates were dried at $60{ }^{\circ} \mathrm{C}$ for $48 \mathrm{~h}$ and their composition was determined as mentioned earlier.

\section{Enzymatic hydrolysis}

Enzymatic saccharification of pretreated and untreated barley straw was carried out at $50{ }^{\circ} \mathrm{C}$ and $150 \mathrm{rpm}$ in a shaker incubator. The cellulase activity was determined based on NERL. Cellulase and $\beta$-glucosidase were loaded in at $40 \mathrm{FPU} \mathrm{g}^{-1}$ substrate and $200 \mathrm{CBU} \mathrm{g}^{-1}$ substrate, respectively. Samples were withdrawn at 3, 6, 12, 24 and $72 \mathrm{~h}$ for analysis. For each vial, $5.0 \mathrm{ml}$ sodium citrate buffer $0.1 \mathrm{M}$ (PH 4.8) was added to the equivalent amount of $0.15 \mathrm{~g}$ total barley straw biomass. Moreover, $40 \mu \mathrm{L}$ $(400 \mu \mathrm{g})$ tetracycline and $30 \mu \mathrm{L}(300 \mu \mathrm{g})$ cycloheximide were also added into each vial to prevent the growth of organisms during the digestion. After addition of the enzymes, the volume of each vial was brought to $10 \mathrm{ml}$ by 
addition of deionized water. Glucose concentration in each vial was determined by high-performance liquid chromatography (HPLC) with an RI detector (Knauer, Germany) equipped with a Eurokat $\mathrm{H}$ carbohydrate analysis column (Knauer, Germany). The mobile phase was acidified waster $(0.01 \mathrm{~N}$ sulfuric acid, $\mathrm{pH} 2$,$) , at a flow rate of 1 \mathrm{ml} \mathrm{m^{-1 }}$ with a column temperature of $65{ }^{\circ} \mathrm{C}$.

\section{FTIR analysis}

The chemical structure of untreated and pretreated barley straw was characterized using Fourier transform infrared (FTIR-FIR) spectrometry (Equinox 55, Bruker Germany). All biomass samples were dried and mixed with potassium Bromide $(\mathrm{KBr})$ before pressing the sample into discs.

\section{Scanning electron microscopy}

The effect of pretreatment on the morphology of the barley straw was observed with scanning electron microscopy (SEM) (TESCAN_VEGA) at an acceleration voltage of $20 \mathrm{kV}$. Samples were mounted on aluminum sample stubs and sputtered with a thin layer of gold. Finally, many spots (at least five) were considered for each sample under different magnifications.

\section{Crystallinity measurement}

X-ray powder diffraction (XRD) (Siemens, Model D5000, Germany) was applied to characterize the crystallinity of lignocellulosic materials for pretreated and untreated barley straw. XRD data were measured at $25{ }^{\circ} \mathrm{C}$ using a $\mathrm{Fe}$ tube (voltage $35 \mathrm{~kW}, 25 \mathrm{~mA}$ ). Samples were scanned over the range of $5^{\circ}-70^{\circ}$ with a step size of 0.02 s and step time of $10 \mathrm{~s}$. Crystallinity index $(\mathrm{Crl})$ was determined based on the XRD data and calculated using the following formula (Segal et al. 1959):

$\mathrm{Crl}=\left(I_{002}-I_{\mathrm{am}}\right) / I_{002}$.

In which, $I_{002}$ is the intensity for crystalline portion of biomass at about $2 \theta=22.5$ and $I_{\mathrm{am}}$ is the peak for the amorphous portion (i.e., cellulose, hemicelluloses and lignin) at about $2 \theta=16.6$. The second highest peak after $2 \theta=22.5$ was $2 \theta=16.6$, and was assumed to correspond to amorphous region (Kumar et al. 2009).

\section{Result and discussion}

The application of ILs has been received much attention recently. ILs as solvents possess some advantages such as no cellulose decomposition, easy processing, easy cellulose regeneration (precipitation upon addition of anti-solvent, e.g. deionized water), and no toxicity (Tam-Anh et al. 2010). In the present study, a wide range of ionic liquids, i.e. 1-ethyl-3-methyl imidazolium acetate, 1-ethyl-3-methyl imidazolium diethyl phosphate, 1-butyl-3-methyl imidazolium chlorides, 1,3-dimethyl imidazolium dimethyl phosphate, and 1-butyl-3-methylimidazolium-trifluoromethane sulfonate were examined in order to find the best solvent for barley straw at $110{ }^{\circ} \mathrm{C}$ for $90 \mathrm{~min}$. The composition of barely biomass, i.e. cellulose, hemicellulose, and lignin, was 40.8, 21.76 and $12.18 \%$, respectively. Changes in the composition of the solid phase promoted by ILs pretreatment were evaluated by measuring cellulose content. The cellulose content after pretreatment by [EMIM][AC], [EMIM][DEP], [MMIM][DMP], [BMIM][CL], and [BMIM][OTf] was recorded at 51.83, 43.10, 42.75, 42.49 and 40.18, respectively. As clearly observed [EMIM][AC] led to the highest compositional change in the barely biomass. These differences could be attributed to the important ionic parameters such as cation and anion size as well as hydrogen bond basicity (Mäki-Arvela et al. 2010). In the present study, $[\mathrm{EMIM}]^{+}$was found of better dissolution capability in comparison with $[\mathrm{BMIM}]^{+}$. This could be explained by the fact that small cations ([EMIM]) are often more efficient in dissolving cellulose than larger ones ([BMIM)] (Kosan et al. 2008). Moreover, the better performance of [MMIM][DMP] compared to [BMIM][CL] and [BMI$\mathrm{M}][\mathrm{OTf}]$ was due to its higher basicity (Mäki-Arvela et al. 2010).

\section{Cellulose digestibility}

After pretreatment and $3 \mathrm{~h}$ hydrolysis, the lowest glucose release was obtained for the untreated barley straw while the highest $\left(1.37 \mathrm{mg}\right.$ glucose $\left.\mathrm{ml}^{-1}\right)$ was attributed to the barley straw pretreated by [EMIM][AC]. At the end of the experiment at $72 \mathrm{~h}$ after the commencement of the hydrolysis process, the highest concentration still belonged to barley straw pretreated by [EMIM] [AC] at $3.95 \mathrm{mg}$ glucose $\mathrm{ml}^{-1}$. The results obtained mark [EMIM][AC] as the best IL pretreatment choice for significantly improving the enzymatic saccharification in comparison with the other four ILs used.

Based on the enzymatic hydrolysis data, cellulose digestibility was calculated as described by NREL/TP-51042629 (Selig et al. 2008). Figure 1 shows cellulose digestibility profiles for untreated barley straw as well as barley straw samples pretreated by five ILs while the same enzyme loading was applied to all samples during the hydrolysis process. Significantly higher saccharification was achieved using [EMIM][Ac]-pretreated barley straw showed with cellulose digestibility reaching $76 \%$ within $72 \mathrm{~h}$, whereas digestibility of untreated barley straw only reached $20 \%$. 
Fig. 1 Comparison of untreated (control) and treated barely samples with different ionic liquids in terms of cellulose digestion $(\%)$ versus time

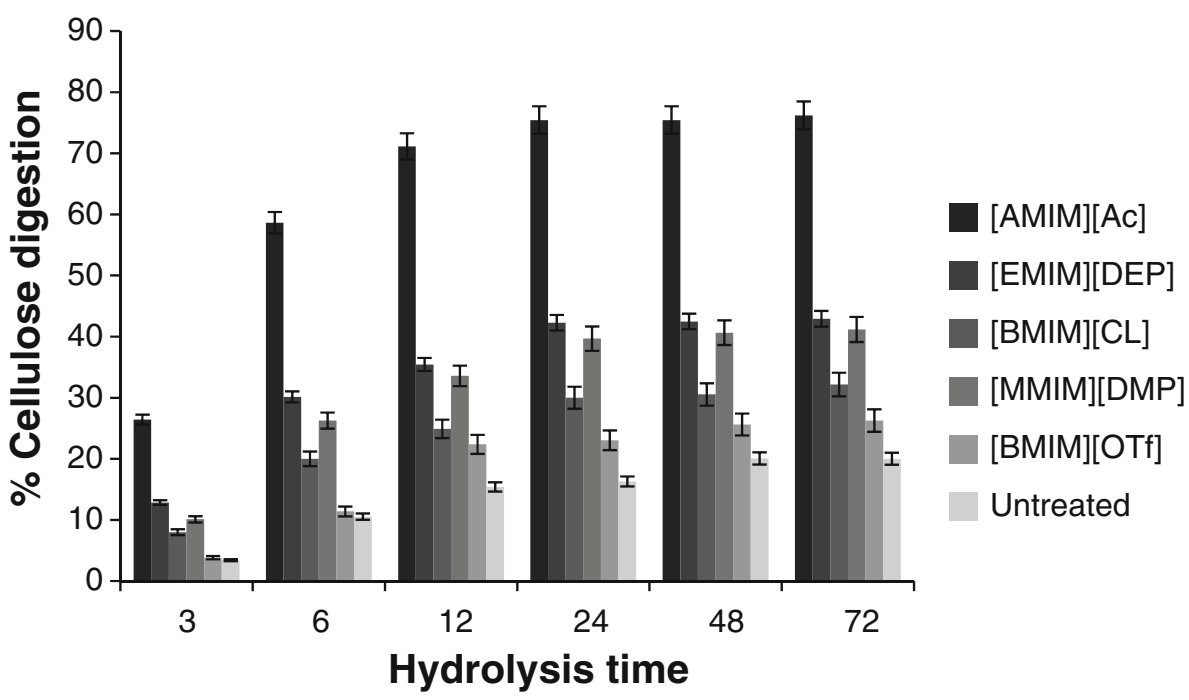

This difference caused by ILs in particular [EMIM][Ac] could be ascribed to the loss of intra- and inter-molecular hydrogen bonds leading to the formation of amorphous cellulose and consequently increased surface area. The latter leads to better enzyme accessibility and increased binding sites in recovered cellulose fibers ( $\mathrm{Li}$ et al. 2010).

\section{FTIR analysis}

The chemical structure of untreated and pretreated barley straw samples was analyzed using FTIR. As shown in Fig. 2 and Table 1, the spectra generated for samples pretreated by ILs were similar to that of the untreated barley straw; however, there were some small differences observed. For instance, at $897 \mathrm{~cm}^{-1}$, the peak obtained was more intense in cases of [EMIM][AC]- and [EMIM][DEP]-pretreated barley straws compared with untreated, [BMIM][CL]-, [MMIM][DMP]-, and [BMIM][OTf]-pretreated barley straw. In the presence of amorphous cellulose, the band at $897 \mathrm{~cm}^{-1}$, which characterizes the $\mathrm{C}-\mathrm{O}-\mathrm{C}$ stretching at $\beta$ 1,4 -glycosidic linkage, is strong and sharp. The peak at $1,430 \mathrm{~cm}^{-1}$ can be assigned to bending vibration of $\mathrm{CH}_{2}$. This band is strong in crystalline cellulose and weak in amorphous cellulose. So, the crystalline cellulose in treated samples by [BMIM][CL], [MMIM][DMP], [BMIM][OTf] and untreated barley straw is more than the samples treated by [EMIM][AC] and [EMIM][DEP].

The results obtained indicate that untreated barley straw contained higher amount of crystalline cellulose. On the other hand, cellulose in barley straw became more amorphous after pretreatment using ILs. It could be concluded that the amount of amorphous cellulose was highest in the barely sample pretreated by [EMIM][AC], followed by [EMIM] [DEP], [BMIM][CL], [MMIM][DMP], and [BMIM][OTf], respectively. The peaks at 1,328 and $1,514 \mathrm{~cm}^{-1}$ were indicators of lignin characteristic. More specifically, $1,328-\mathrm{cm}^{-1}$ peak reveals the aromatic hydroxyl groups generated by the cleavage of ether bonds within lignin whereas that of $1,514 \mathrm{~cm}^{-1}$ is associated with the aromatic skeletal modes of lignin (Hsu et al. 2010).

As observed in Fig. 2 and Table 1, barley straw samples subjected to IL pretreatment were delignified slightly for the peaks generated at 1,328 and $1,514 \mathrm{~cm}^{-1}$ were identical and that there was a subtle difference between the ILs pretreated samples and the untreated one. However, barley straw subjected to [EMIM][DEP] pretreatment was delignified slightly more efficiently in comparison with the other IL pretreatments. Overall as could be concluded from Fig. 2 and Table 1, using ILs is not a suitable method for removing lignin.

\section{Scanning electron microscopy}

Figure 3 presents the physical structural changes obtained in barley straw during the ILs pretreatment. SEM images of untreated and ILs-pretreated barley straw samples were taken at $500 \times, 1,000 \times$ and $3,000 \times$ magnifications. The results obtained indicate that the untreated barley straw had a highly fibrillar and intact morphology (Fig. 3a-c) in comparison with those that underwent IL-pretreatments (Fig. 3d-r). Among the ILs used, [EMIM][AC] pretreatment was clearly proven to have altered the structure of barley straw the most (Fig. 3d-f). As shown, the surface has become swollen and loose and the original fibrous structure has been completely distorted after the pretreatment by [EMIM][AC]. In other words, the fibrous structure of the barely straw has been transformed into a porous and amorphous form after the [EMIM][AC] pretreatment.

[EMIM][DEP] and [MMIM][DMP] pretreatments had similar effects on barley straw (Fig. $3 \mathrm{~g}-1$ ) and led to maximum alterations in barley straw structure after 
Fig. 2 The FTIR spectra of barley samples pretreated by different ionic liquids

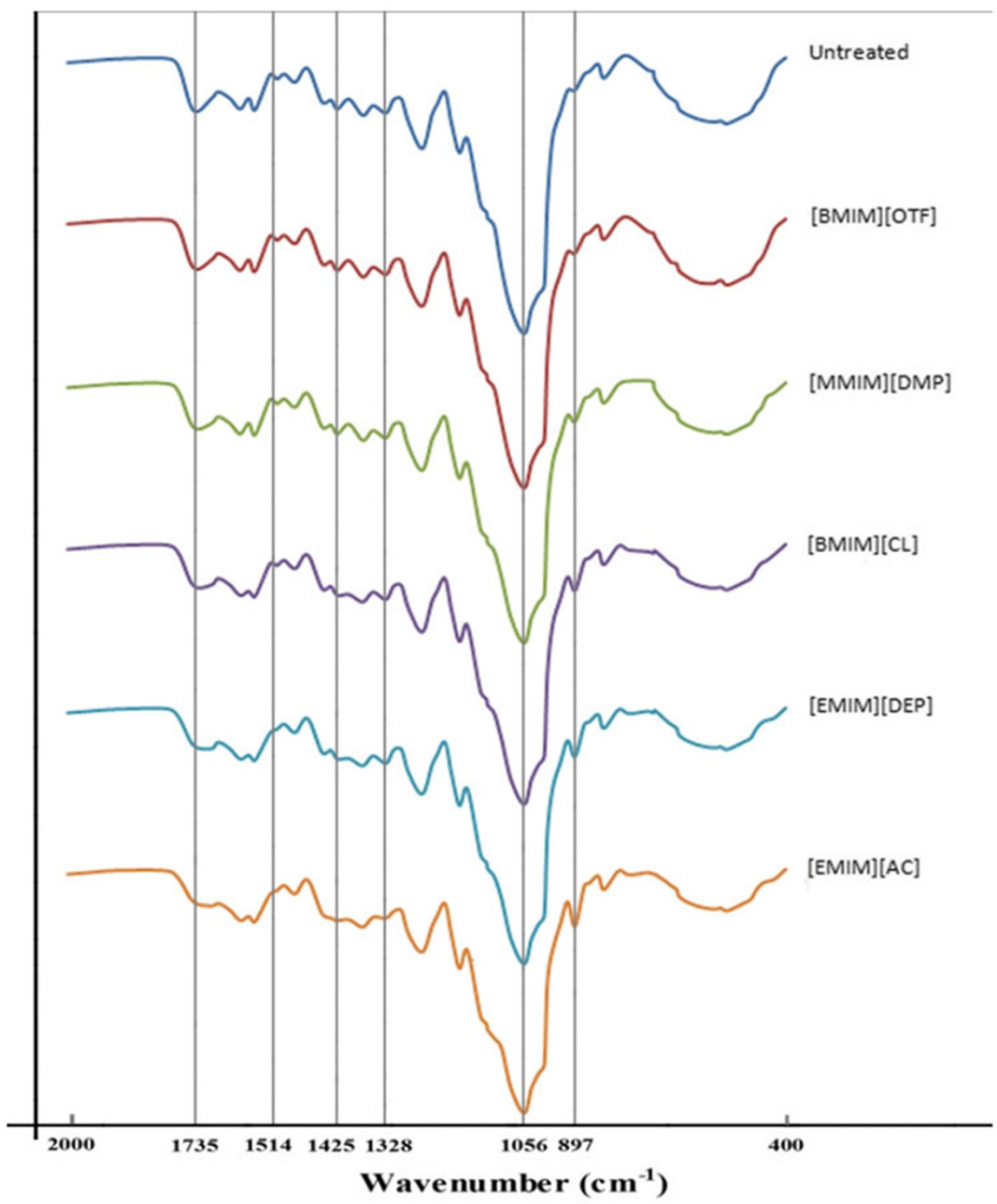

Table 1 FTIR intensity values obtained for barley samples pretreated by different ionic liquids

\begin{tabular}{|c|c|c|c|c|c|c|}
\hline \multirow[t]{2}{*}{ Treatment } & \multicolumn{6}{|c|}{ FTIR peaks $\left(\mathrm{cm}^{-1}\right)$} \\
\hline & 897 & 1,056 & 1,328 & 1,425 & 1,514 & 1,735 \\
\hline Untreated & 91.020 & 96.810 & 91.557 & 91.230 & 90.652 & 91.494 \\
\hline$[\mathrm{BMIM}][\mathrm{OTF}]$ & 91.054 & 96.630 & 91.554 & 91.231 & 90.655 & 91.420 \\
\hline [MMIM][DMP] & 91.155 & 96.425 & 91.556 & 91.232 & 90.652 & 91.302 \\
\hline [BMIM][CL] & 91.343 & 96.404 & 91.574 & 91.240 & 90.707 & 91.436 \\
\hline [EMIM][DEP] & 91.405 & 96.323 & 91.564 & 91.247 & 90.838 & 91.127 \\
\hline [EMIM][AC] & 91.590 & 96.026 & 91.388 & 91.251 & 90.848 & 91.033 \\
\hline
\end{tabular}

[EMIM][AC] pretreatment. On the ILs list, the less effect on barley straw physical structure belonged to [BMI$\mathrm{M}][\mathrm{OTf}]$ pretreatment which was incapable of making any significant alterations (Fig. 3p-r).
Barley straw crystallinity

Biomass crystallinity is an important feature affecting enzymatic hydrolysis. Different pretreatment methods can 

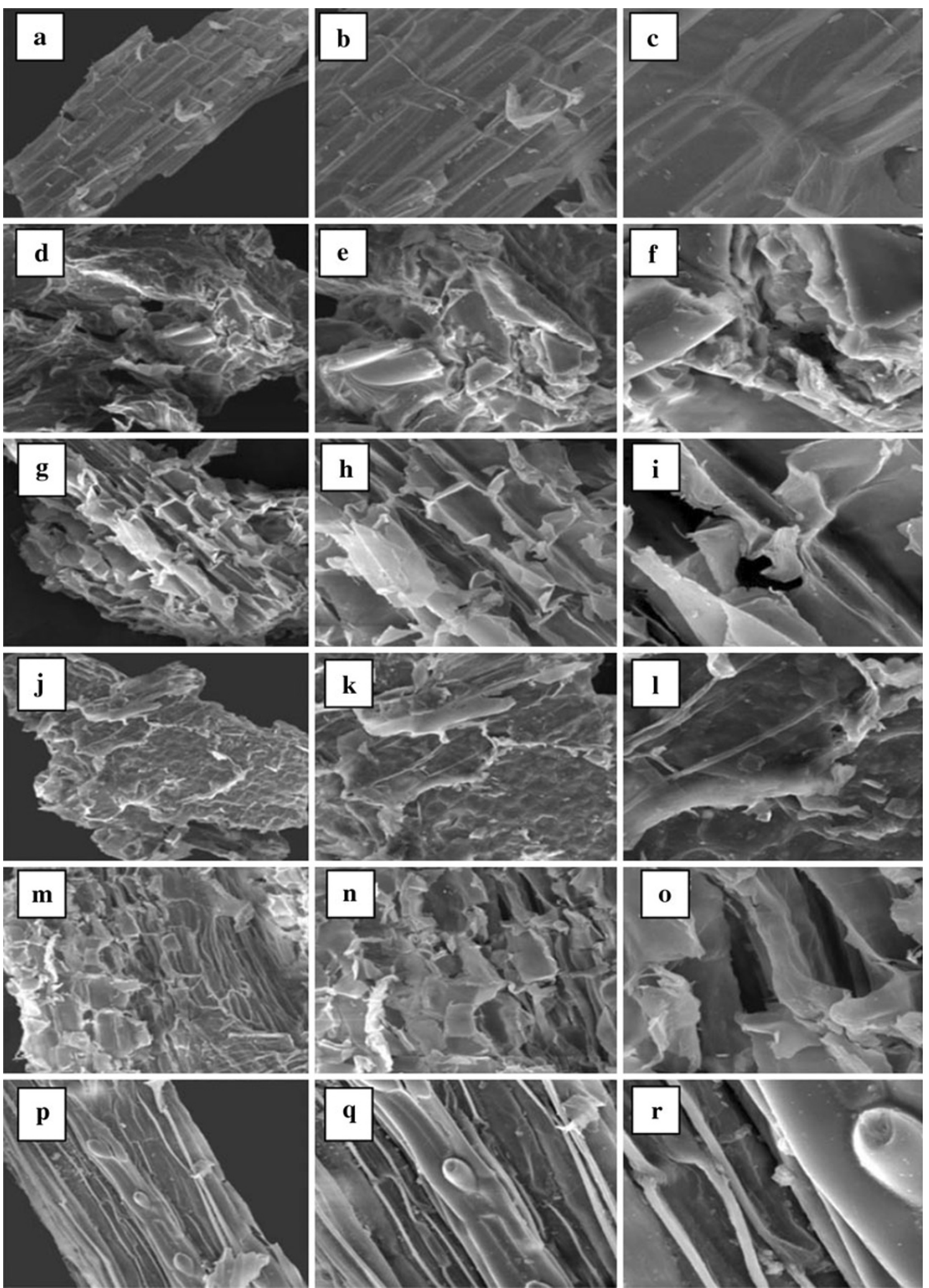

Fig. 3 SEM images of barley straw; a-c raw barley straw $(\times 500$, $\times 1,000, \times 3,000)$; d-f [EMIM][AC]-pretreated barley straw $(\times 500$, $\times 1,000, \times 3,000) ; \quad$ g-i $\quad[$ EMIM][DEP]-pretreated barley straw $(\times 500, \times 1,000, \times 3,000) ; \mathbf{j}-\mathbf{l}[\mathrm{BMIM}][\mathrm{CL}]$-pretreated barley straw $(\times 500, \times 1,000, \times 3,000) ; \mathbf{m}-\mathbf{o}[\mathrm{MMIM}][\mathrm{DMP}]-$ pretreated barley straw $(\times 500, \times 1,000, \times 3,000)$; $\mathbf{p}-\mathbf{r}$ [BMIM] [OTf] pretreated barley straw $(\times 500, \times 1,000, \times 3,000)$ 
alter cellulose crystal structures by disrupting inter- and intra-chain hydrogen bonding of cellulose fibrils (Mosier et al. 2005). In this study, the features of regenerated barley straw samples after various IL pretreatments were examined using X-ray diffraction and were also compared to those of untreated barley straw. Untreated barley straw was found highly crystalline $(59.5 \mathrm{Crl})$. After [EMIM][AC] pretreatment, $\mathrm{Crl}$ index of barley straw was decreased significantly to 15.2 revealing minimal structural order in cellulose after the pretreatment. This Crl value was the least when compared with those achieved through the application of the other ILs in the pretreatment process. In other words, this sharp decrease in crystallinity due to the [EMIM][AC] pretreatment confirms that the regenerated products were highly amorphous and thus, cellulose surface accessibility and consequently the efficiency of enzymatic hydrolysis were considerably increased. After [EMIM][DEP], [BMIM] [CL], [MMIM][DMP], and [BMIM][OTf] pretreatments, Crl value of barley straw was decreased to $30,48,30.5$, and 57 , respectively.

\section{Conclusion}

Due to the worldwide cultivation of barley, its straw is one of the most important feedstock for the production of fermentable sugar and bioethanol. Among the 5 different ILs examined, [EMIM][AC] was found to have led to the highest degree of highest cellulose conversion. The SEM, FTIR, and XRD analyses ranked [EMIM][AC] pretreatment followed by [EMIM][DEP] pretreatment as most efficient in terms of altering the physical structure of barley straw. Overall, [EMIM][Ac]-pretreated barley straw showed significantly higher saccharification with cellulose digestibility reaching $76 \%$ after $72 \mathrm{~h}$, whereas digestibility of untreated barley straw only reached $20 \%$.

Acknowledgments The authors would like to thank Biofuel Research Team (BRTeam) and Agricultural Biotechnology Research Institute of Iran (ABRII) for financing this project.

Open Access This article is distributed under the terms of the Creative Commons Attribution License which permits any use, distribution, and reproduction in any medium, provided the original author(s) and the source are credited.

\section{References}

Alvira P, Tomás-Pejó E, Ballesteros M, Negro MJ (2010) Pretreatment technologies for an efficient bioethanol production process based on enzymatic hydrolysis: a review. Bioresour Technol 101:4851-4861
Chandra RP, Bura R, Mabee WE, Berlin A, Pan X, Saddler J (2007) Substrate pretreatment: the key to effective enzymatic hydrolysis of lignocellulosics. Adv Biochem Eng Biotechnol 108:67-93

Galbe M, Zacchi G (2007) Pretreatment of lignocellulosic materials for efficient bioethanol production. Adv Biochem Eng Biotechnol 108:41-65

Guragain YN, Coninck JD, Husson F, Durand A, Rakshit SK (2011) Comparison of some new pretreatment methods for second generation bioethanol production from wheat straw and water hyacinth. Bioresour Technol 102:4416-4424

Haghighi Mood S, Golfeshan AH, Tabatabaei M, Salehi Jouzani Gh, Najafi Gh, Gholami M, Ardjmand M (2013) Lignocellulosic biomass to bioethanol; a comprehensive review on pretreatment. Renew Sust Energ Rev 27:77-93

Hsu TC, Guo GL, Chen WH, Hwang WS (2010) Effect of dilute acid pretreatment of rice straw on structural properties and enzymatic hydrolysis. Bioresour Technol 101:4907-4913

Kosan B, Michels C, Meister F (2008) Dissolution and forming of cellulose with ionic liquids. Cellulose 15:59-66

Kumar R, Mago G, Balan V, Wyman CE (2009) Physical and chemical characterizations of corn stover and poplar solids resulting from leading pretreatment technologies. Bioresour Technol 100:3948-3962

Li Q, He YC, Xian M, Jun G, Xu X, Yang JM, Liang-Zhi L (2009) Improving enzymatic hydrolysis of wheat straw using ionic liquid 1-ethyl-3-methyl imidazolium diethyl phosphate pretreatment. Bioresour Technol 100:3570-3575

Li C, Knierim B, Manisseri C, Arora R, Scheller HV, Auer M, Vogel KP, Simmons BA, Singh S (2010) Comparison of dilute acid and ionic liquid pretreatment of switchgrass: biomass recalcitrance, delignification and enzymatic saccharification. Bioresour Technol 101:4900-4906

Mäki-Arvela P, Anugwom I, Virtanen P, Sjöholm R, Mikkola JP (2010) Dissolution of lignocellulosic materials and its constituents using ionic liquids - a review. Ind Crop Prod 32:175-201

Mora-Pale M, Meli L, Doherty TV, Linhardt RJ, Dordick JS (2011) Room temperature ionic liquids as emerging solvents for the pretreatment of lignocellulosic biomass. Biotechnol Bioeng 108:1229-1245

Mosier N, Wyman C, Dale B, Elander R, Lee YY, Holtzapple M, Ladisch M (2005) Features of promising technologies for pretreatment of lignocellulosic biomass. Bioresour Technol 96:673-686

Segal L, Creely JJ, Martin AE Jr, Conrad CM (1959) An empirical method for estimating the degree of crystallinity of native cellulose using the X-ray diffractometer. Text Res J 29:786-794

Selig M, Weiss N, Ji Y (2008) Enzymatic saccharification of lignocellulosic biomass: laboratory analytical procedure (LAP), NREL/TP-510-42629. National Renewable Energy Laboratory (NREL)

Sluiter A, Hames B, Ruiz R, Scarlata C, Sluiter J, Templeton D, Crocker D (2008) Determination of structural carbohydrates and lignin in biomass: Laboratory Analytical Procedure (LAP), NREL/TP-510-42618, National Renewable Energy Laboratory (NREL)

Tam-Anh DN, Kyoung-Rok K, Se JH, Hwa YC, Jin Woo K, Sung Min Park C, Jae Chan Park C, Sang Jun S (2010) Pretreatment of rice straw with ammonia and ionic liquid for lignocelluloses conversion to fermentable sugars. Bioresour Technol 101: $7432-7438$

Tan HT, Lee KT, Mohamed AR (2010) Pretreatment of lignocellulosic palm biomass using a solvent-ionic liquid [BMIM]Cl for glucose recovery: an optimisation study using response surface methodology. Carbohyd Polym 83:1862-1868 
Wyman CE, Dale BE, Elander RT, Holtzapple M, Ladisch MR, Lee YY (2005) Coordinated development of leading biomass pretreatment technologies. Bioresour Technol 96:1959-1966

Yoon LW, Ngoh GC, Chua ASM, Hashim MA (2011) Comparison of ionic liquid, acid and alkali pretreatments for sugarcane bagasse enzymatic saccharification. J Chem Technol Biotechnol 86: $1342-1348$

Zhao H, Jones CL, Baker GA, Xia S, Olubajo O, Person VN (2009) Regenerating cellulose frome ionic liquids for an accelerated enzymatic hydrolysis. Green Chem 8:325-327 